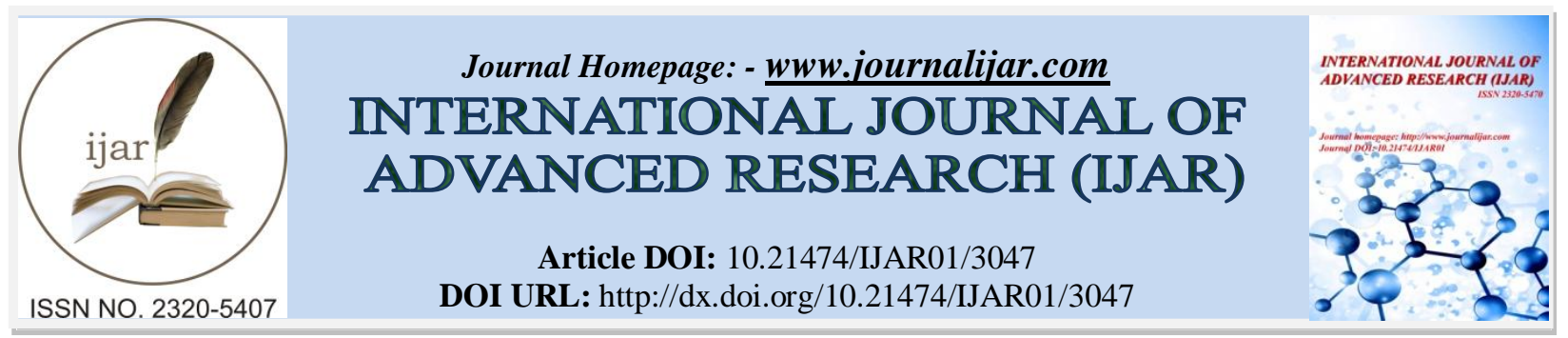

RESEARCH ARTICLE

\title{
ROLE OF WEIGHT REDUCTION VERSUS PHYSICAL THERAPY IN MANAGEMENT OF OBESE PATIENTS WITH KNEE OSTEOARTHRITIS.
}

\author{
Heba A. Seliem ${ }^{1}$, Eman E. El-Shahawy ${ }^{1}$, Samah F. Abbas ${ }^{1}$ and Asmaa A.Baz ${ }^{2}$. \\ 1. Department of Rheumatology and Rehabilitation, Faculty of medicine Zagazig University. \\ 2. Ministery of Health ,Egypt.
}

\section{Manuscript Info}

.........................

Manuscript History

Received: 30 November 2016

Final Accepted: 28 December 2016

Published: January 2017

\section{Abstract}

Objective: the aim of our work was to study the effects of physical therapy versus weight reduction and their combined effects in obese patients with knee OA.

Methods: 156 obese patients (137 females and 19 males) with knee OA were recruited. their BMIs ranged from 30.2 to 50.8. Patients were randomized to three groups: Group I: underwent physical therapy only. Group II: underwent weight reduction only. Group III: underwent combined physical therapy and weight reduction. Changes in body weight and body composition were examined as independent predictors of changes in knee OA symptoms. Symptoms were monitored by the Western Ontario and McMaster Universities' (WOMAC) OA index.

Results: the mean changes of group II and III (-16.9 \pm 2.7 and -15.7 \pm 2 ) were highly significant respectively while mean change of group I $(-0.6 \pm 0.4)$ was non significant. Group II had the best improvement of BMI with mean change of $(-6.6 \pm 0.8)$ followed by group III $(-4.9 \pm 0.7$ ) while group I had the worst $(-0.3 \pm 0.2)$. The total WOMAC index improved within each group after two and four months from baseline ( $\mathrm{p}<0.001$ ), The best mean change of WOMAC score was found in group III $(-14.8 \pm 49)$.

Conclusion: Combination of both weight reduction and physical therapy gives better results as regard improving knee pain and function in patients with $\mathrm{OA}$, reduces disability and improves quality of life compared to each method alone

Copy Right, IJAR, 2016,. All rights reserved.

\section{Introduction:-}

$\mathrm{OA}$ is the most common cause of musculoskeletal disability in the elderly, and it places an enormous economic burden on society (1).

Osteoarthritis (OA) of the knee is a degenerative joint disease with progressive degradation of articular cartilage and subchondral bone. Clinical manifestations may include joint pain, tenderness, stiffness, locking and joint effusion depending on the stage of the disease (2).

Obesity is one of the most significant and potentially most preventable risk factor for the development of OA and numerous studies have shown a strong association between body mass index and OA of the knee (3). 
Obesity and knee OA are two of the most common chronic diseases and are often co-morbid. The risk of knee OA related to weight gain and obesity begins from an early age. Weight loss reduces the risk of incident knee OA and, in established disease, reduces symptoms, improves function and is likely to reduce disease progression (4).

The precise mechanism by which obesity leads to OA remains unknown, but is likely to be due to a combination of mechanical, humoral and genetic factors. Weight loss has clear medical benefits for the obese patient and seems to be a logical way of relieving joint pain associated with degenerative arthritis (5).

Exercise therapy reduces pain and activity limitations in OA of the knee. Classical exercise therapy is aimed at improving muscle strength, aerobic capacity, range of joint motion, and training of walking and activities of daily living (6).

Weight loss, preferably combined with exercise therapy, reduces pain and activity limitations in OA patients who are overweight (6).

Aim of the work:-

The aim of the work was to study the effects of physical therapy versus weight reduction and their combined effects in obese patients with knee osteoarthritis

\section{Patients and Methods:-}

This study was carried out at rheumatology and rehabilitation department at Zagazig university hospitals, Egypt on 156 obese patients (137 females and 19 males) aged between 38 and 57 years old with disease duration of 1 to 9 years of primary knee OA diagnosed according to The American College of Rheumatology (ACR) classification criteria of OA (7).

After taking a written consent from them for ethical consideration, patients were randomly divided into three groups: Group I: They underwent physical therapy only. Group II: They underwent weight reduction only. Group III: They underwent combined physical therapy and weight reduction. All patients in this study had radiographic severity grade I or II according to the Kellegren-Lawrence grading system (8).

Major exclusion criteria were: Other rheumatic diseases that might be responsible for 2ry OA, haematological disorders., CVS, renal or hepatic diseases, DM or other endocrinal disorders, patients having grade III andIV knee OA or history of knee trauma. Before inclusion in the study, fasting blood glucose was measured as well as hemoglobin and TSH. All patients meeting the previously mentioned inclusion criteria were initially assessed at 1 st visit by full medical history, general and systemic examination, Local examination of both knees, assessment of pain severity using visual analogue scale (VAS). (9), measuring functional disability using WOMAC questionnaire (Western Ontario and McMaster Universities Arthritis Index) (10), determination of ambulation activity by measuring the walking speed (time needed (in seconds) to complete a distance of 100 meters on a treadmill as tolerably and quickly as possible) (11), measuring body mass index (BMI) for all patients included in the study using the following equation : $\mathrm{BMI}=($ Weight $($ in $\mathrm{Kgs}) /$ Height $($ in meters $)(\mathbf{1 2})$.

Calculating Estimated Nutrient Needs: Nutritional needs were calculated for patients in group II and III according to Harris Benedict Equation which calculates the resting energy expenditure (REE) (13) and recalculated every 2 weeks to change diet program.

\section{Physical therapy programs:-}

Physical therapy programs were done for patients in group I and III in the form of TENS, quadriceps exercise, ultrasonic, cold packs, infra-red or faradic stimulation. These modalities were chosen according to severity of symptoms and reevaluated every 2 weeks to change the program.

\section{Follow up sessions:-}

All patients in this study were reevaluated after 2 and 4 months by: Full medical history and physical examination, reassessment of VAS scale and WOMAC index, reassessment of ambulation activity by measuring walking speed and reassessment of BMI. 


\section{Statistical analysis:-}

The statistical analysis of data was done by using SPSS program (statistical package for social science) version 20.0. The description of the data was done in form of mean and standard deviation for quantitative data, frequency and proportion for qualitative data. For quantitative data one way ANOVA test was used to compare the means of the three groups. Repeated measure ANOVA test was used for the comparison of the means of each group at the three readings during the follow up. For qualitative data (frequency and proportion), Chi- square test was used. The correlations between the quantitative variables was tested using the Pearson correlation test.

The mean change was calculated by calculating the difference between the mean of the variable after the intervention and the mean of the variable at the baseline. All data were tested for normality of distribution prior to nay calculations. Statistical significant difference was considered at $\mathrm{P}<0.05$, and highly significant difference at $\mathrm{P}<0.001$.

\section{Results:-}

The study was a clinical trial conducted on 156 cases with disease duration of 1 to 9 years of primary knee OA who were divided into three groups, each of which included 52 cases. Seven cases were dropped during the study (4 refused to continue and 3 had depression). Finally, we had 149 patients divided into 3 groups; 50 patients in group I (44 females and 6 males) aged between 38-52 years who underwent physical therapy only, 50 patients in group II (45 females and 5 males) aged between 38-57 years who underwent weight reduction only using weight reduction program and 49 patients in group III (44 females and 5 males) aged between 38-55 years who underwent combined physical therapy and weight reduction (using same weight reduction program).

Table 1:- Characteristics of the studied groups at baseline.

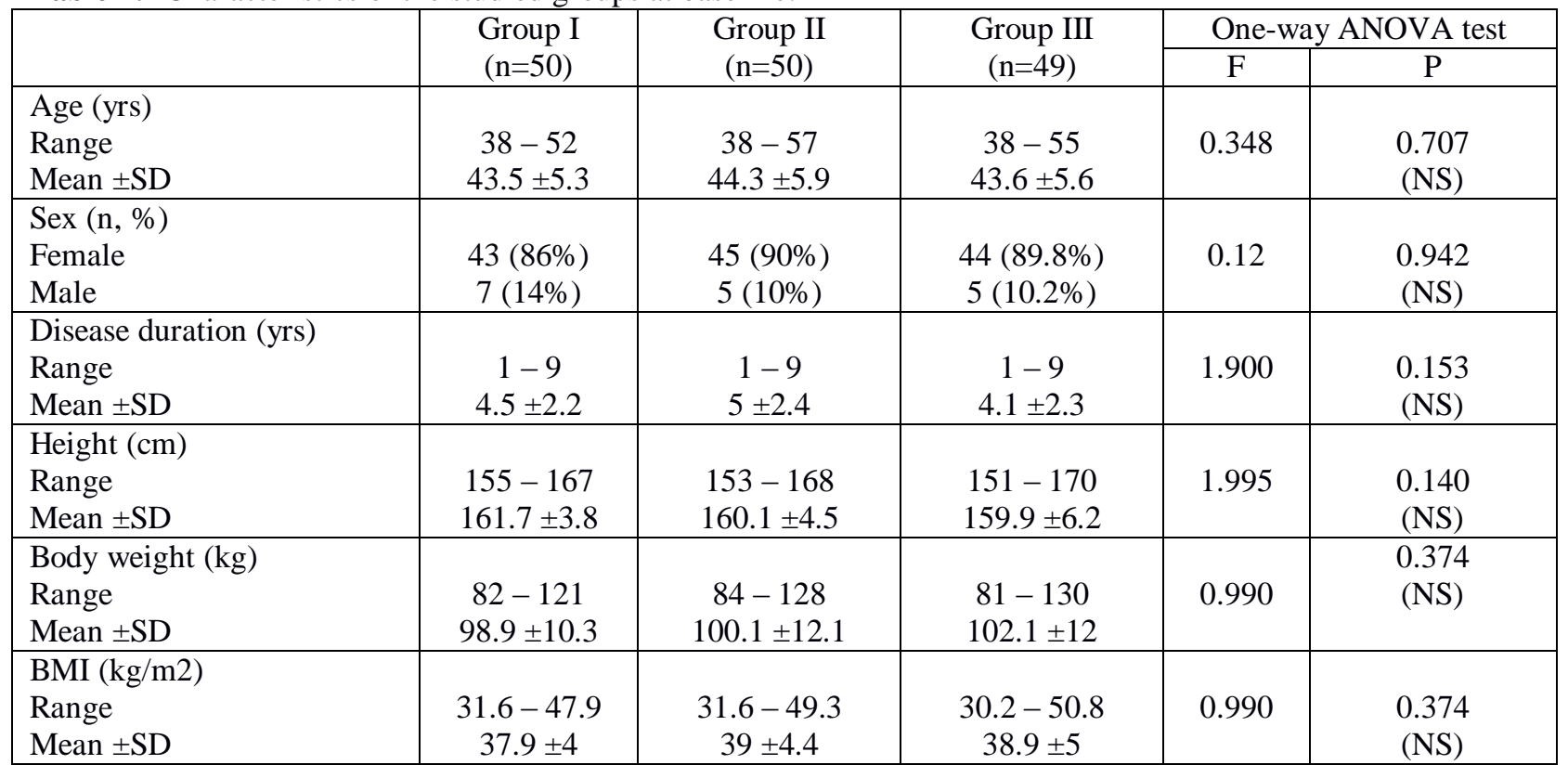

Table 2:- Pain and functional scores of the studied groups at baseline.

\begin{tabular}{|l|c|c|c|c|c|}
\hline & $\begin{array}{c}\text { Group I } \\
(\mathrm{n}=50)\end{array}$ & $\begin{array}{c}\text { Group II } \\
(\mathrm{n}=50)\end{array}$ & \multicolumn{2}{c|}{$\begin{array}{c}\text { Group III } \\
(\mathrm{n}=49)\end{array}$} & \multicolumn{2}{|c|}{ One-way ANOVA test } \\
\cline { 5 - 6 } & & & & $\mathrm{F}$ & $\mathrm{P}$ \\
\hline WOMAC & $6-40$ & $9-43$ & $16-44$ & 0.493 & 0.612 \\
Range & $28.6 \pm 7.8$ & $28 \pm 8.9$ & $29.6 \pm 7.9$ & & $(\mathrm{NS})$ \\
Mean \pm SD & & & & 1.467 & \\
\hline VAS scale & $3-7$ & $4-7$ & $5-7$ & & 0.234 \\
Range & $5.7 \pm 0.9$ & $6 \pm 1$ & $5.9 \pm 0.8$ & & $(\mathrm{NS})$ \\
Mean \pm SD & & & & & \\
\hline Walking speed & $80.7-92.3$ & $78.8-100.4$ & $80.8-105.7$ & 2.983 & 0.054 \\
Range & $85.8 \pm 3.1$ & $85.4 \pm 5$ & $87.6 \pm 6.1$ & & $(\mathrm{NS})$ \\
Mean \pm SD & &
\end{tabular}


Table 3:- Correlation between change of body weight and WOMAC score, VAS-pain score and walking speed in the group I of patients.

\begin{tabular}{|l|c|c|}
\hline & \multicolumn{2}{|c|}{ Body weight } \\
\hline & $\mathrm{R}$ & $\mathrm{P}$ \\
\hline WOMAC score & 0.136 & 0.337 (NS) \\
\hline VAS scale & -0.086 & 0.545 (NS) \\
\hline Walking speed & 0.200 & 0.155 (NS) \\
\hline
\end{tabular}

Table 4:- Correlation between change of the body weight and WOMAC score, VAS-pain score and walking speed in group II of patients.

\begin{tabular}{|c|c|c|}
\hline & \multicolumn{2}{|c|}{ Body weight } \\
\hline & $\bar{R}$ & $\mathrm{P}$ \\
\hline WOMAC score & 0.327 & $0.018^{*}$ \\
\hline VAS scale & 0.275 & $0.048^{*}$ \\
\hline Walking speed & 0.279 & $0.045^{*}$ \\
\hline
\end{tabular}

Table 5:- Correlation of the change of the body weight with WOMAC score, VAS scale and walking speed in group III of patients

\begin{tabular}{|l|c|c|}
\hline \multirow{2}{*}{} & \multicolumn{2}{|c|}{ Body weight } \\
\cline { 2 - 3 } & $\mathrm{R}$ & $\mathrm{P}$ \\
\hline WOMAC score & 0.362 & $0.008^{*}$ \\
\hline VAS scale & 0.328 & $0.018^{*}$ \\
\hline Walking speed & 0.343 & $0.013^{*}$ \\
\hline
\end{tabular}

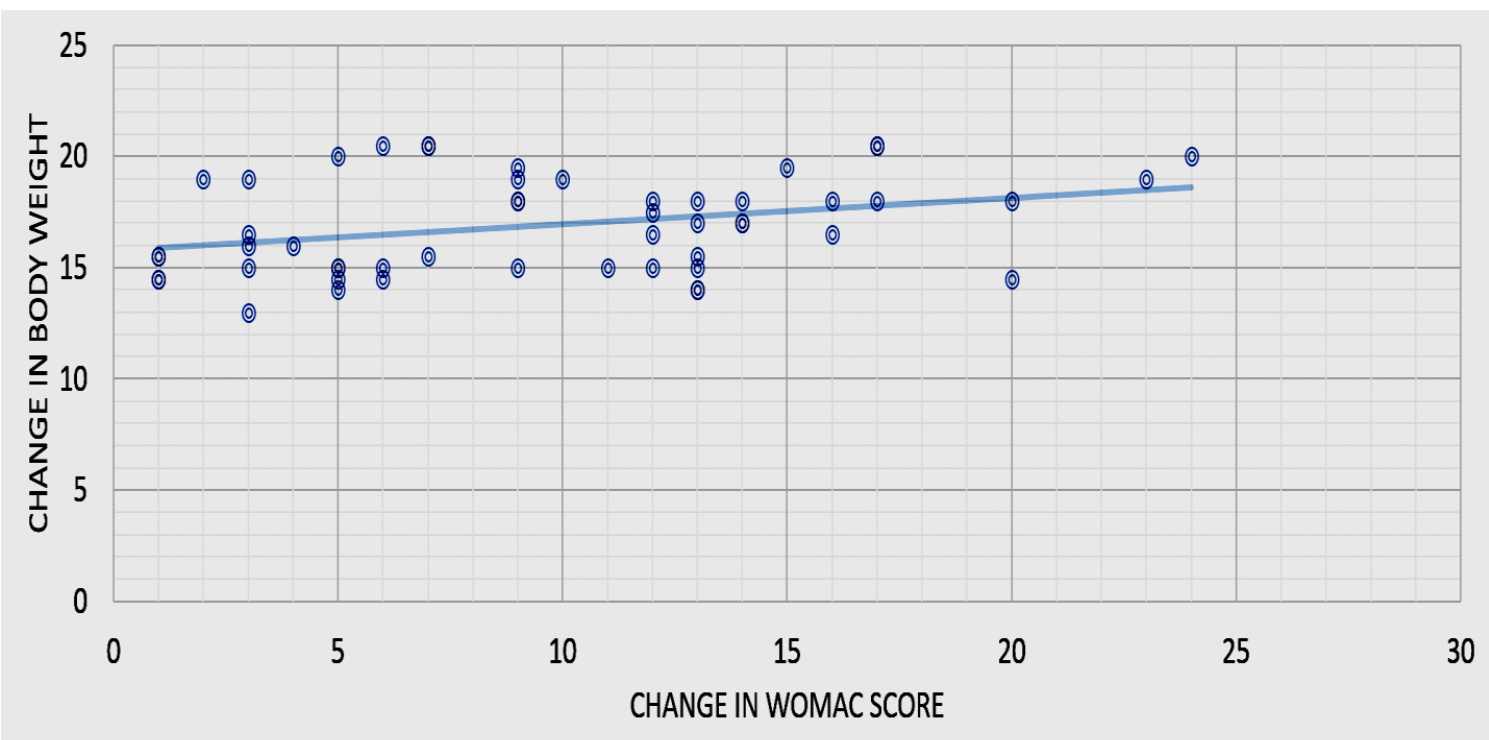

Fig 1:- Correlation between the change of body weight with the change of WOMAC score in group II of patients. 


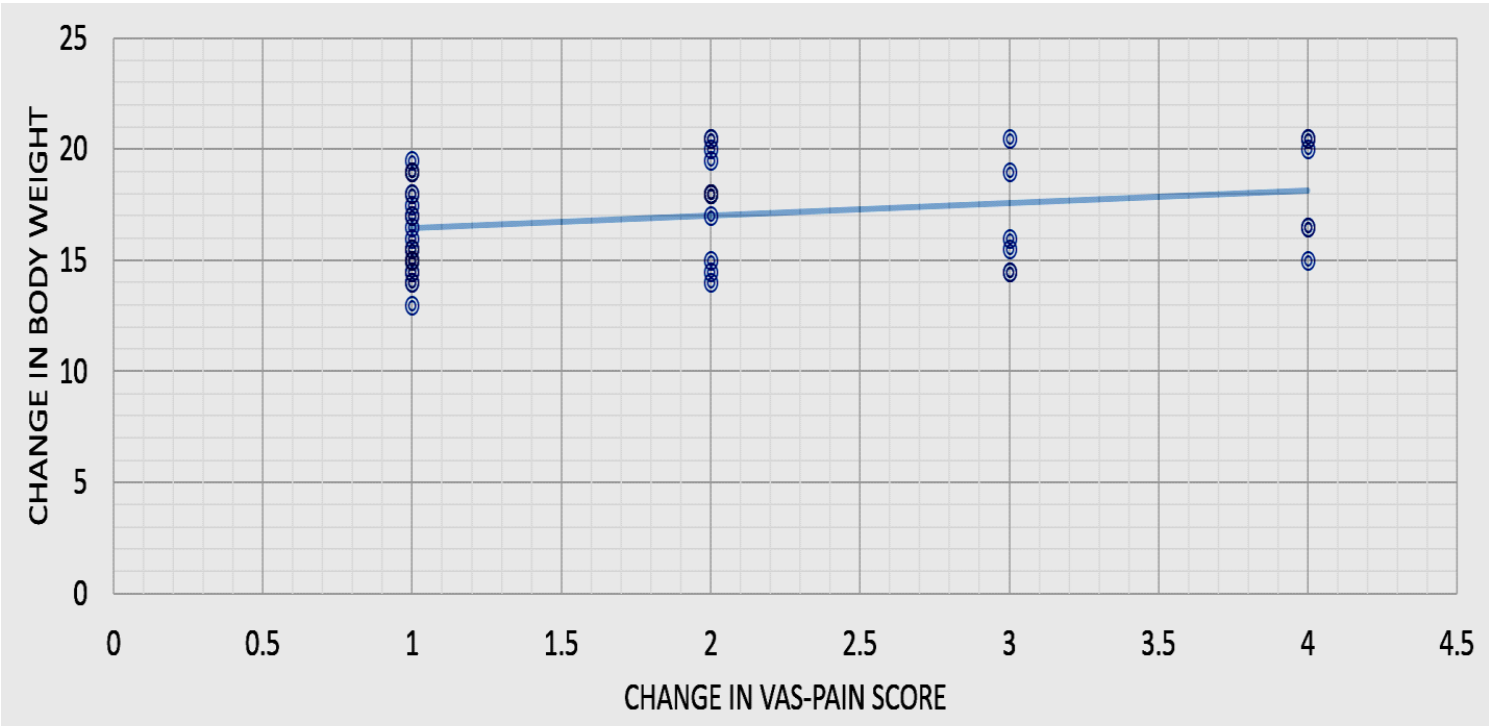

Fig 2:- Correlation between the change of body weight with the change of VAS-pain score in group II of patients.

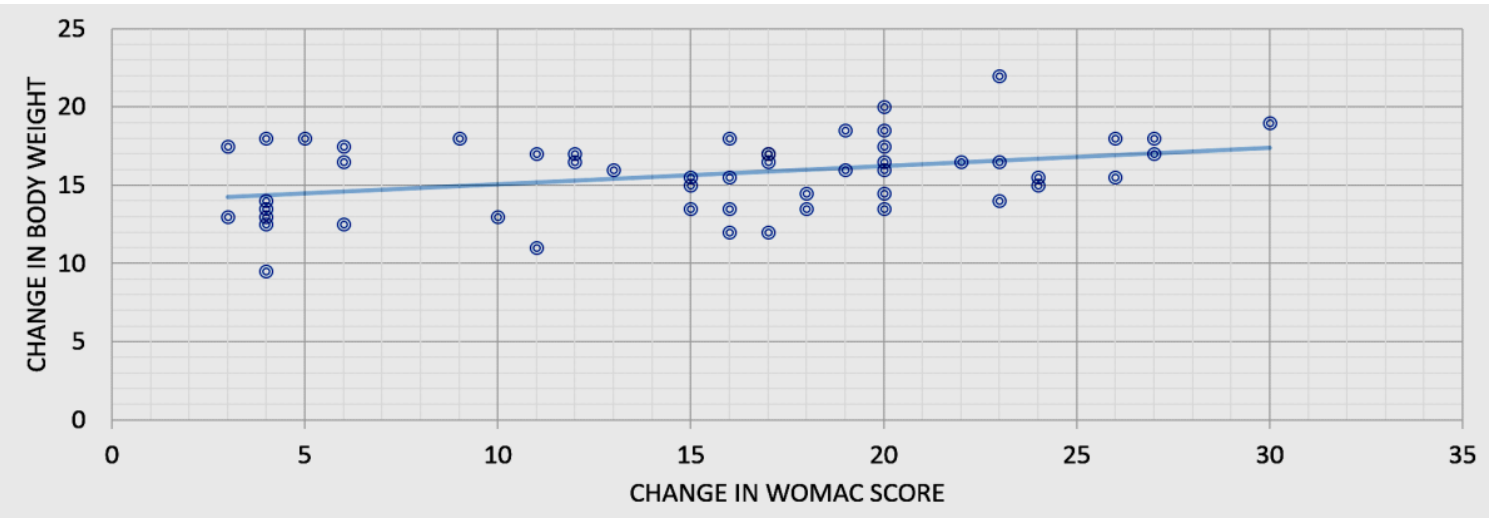

Fig 3:- Correlation between the change of body weight with the change of WOMAC score in group III of patients

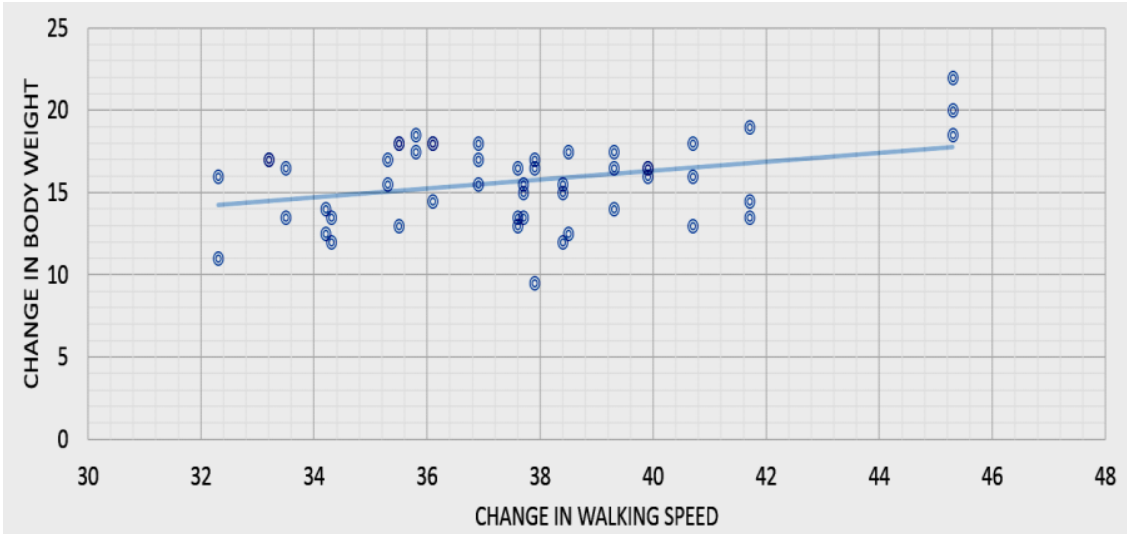

Fig 24:- Correlation between the change of the body weight with the change of walking speed in group III of

\section{Discussion:-} patients.

This study compared the efficacy of physical therapy, weight reduction and their combined effects on 149 obese patients (132 females and 17 males) aged between 38-57 years complaining for 1 to 9 years of primary knee OA. 
Patients were divided into 3 groups; group I underwent physical therapy only, group II underwent weight reduction only and group III underwent both physical therapy and weight reduction.

At the beginning of this study as shown in table 1, body weights of the studied group ranged from 81 to $130 \mathrm{~kg}$, their BMIs ranged from 30.2 to 50.8 while their heights ranged from 151 to $170 \mathrm{~cm}$.

Among all patients, 64 patients had grade I knee OA while 85 patients had grade II. Also, 79 patients had quadriceps wasting. Most of patients (113 patients) had tender joint line while only 53 patients had deformity. Effusion was absent in 64 patients, mild in 55 patients and moderate in 30 patients only.

Regarding pain and functional scores of studied groups at the beginning of the study shown in table 2, WOMAC scores ranged from 6 to 44, VAS score ranged from 3 to 7 and walking speeds ranged from 78.8 to 105.7 seconds.

A special equation (Harris -Benedict equation), (13) was used to calculate nutritional needs for patients undergoing weight reduction in group II and III. Also, different modalities of physical therapy were used including TENS, quadriceps exercise, ultrasonic, cold packs, infrared and faradic stimulation which were chosen according to severity of symptoms for patients in group I and III.

In the present study, there was no significant difference between patients in all groups regarding their demographic data, pain and functional scores, clinical signs and radiographic grades of OA at baseline.

In this study, there was no significant difference between the three groups at baseline as regard body weight and BMI while highly significant difference was present between the three groups after 2 and 4 months. Highly significant difference was present regarding body weights and BMI of patients within group II and group III after 4 months while no significant difference was found in group I. Group II had the best improvement of body weight and BMI followed by group III while group I had the least as detected by comparing the mean change of the 3 groups after 4 months .

These results agreed with Huang et al., (11) who classified 126 obese osteoarthritic patients into 3 groups. Group (a) underwent weight reduction, group (b) underwent electrotherapy and weight reduction while group (c) underwent electrotherapy only. They reported significant change of body weight after 6 months in group (a) and (b). But, they used different protocol than this study as they used triple regimen for weight reduction including auricular acupuncture, diet control and aerobic exercises.

Also a study by Lee and kean, (14) was in agreement with this study. They found significant difference of body weight among the studied groups in their meta analysis on a total number of 36 different studies on obese patients with knee OA. They all used diet control with exercise program to achieve weight reduction.

In the present study, highly significant difference was found regarding WOMAC, VAS and walking speed among the three groups at the end of the study from baseline. Improvement was better in group III followed by group II while group I had the least improvement as detected by comparing the mean change of WOMAC, VAS and walking speed of the three groups at the end of the study.

Results of the present study were in agreement with the results of Huang et al., (11).They used VAS and walking speed similar to us but they used different 112 functional index (lequesne index) to assess functional disability. They had significant difference of pain and functional indices at the end of their study in those groups who underwent combined weight reduction and physical therapy better than the other groups who underwent one method alone.

Results of the present study agreed also with the results of Christensen et al., (15). They also used Lequesne index to assess knee function while walking speed and VAS was used to assess knee pain. They found significant difference as regard knee pain and functional measures in patients who did both weight reduction and physical therapy better than patients that underwent one modality alone.

In the current study, change of body weight was significantly correlated with change of WOMAC score, VAS pain score and improvement of walking speed among patient in groups II and III as shown in tables 4 and 5 while no significant correlation was found in group I regarding all previously mentioned parameters as shown in table 3. 
The study of Huang et al., (11) was in agreement with results of the current study as they found that weight reduction was a practical adjuvant treatment in the rehabilitation of patients with knee OA. They suggested that the amount of weight reduction must be greater than $15 \%$ of the initial body weight to provide effective treatment of joint pain. Furthermore, if the weight reduced were more than $12 \%$ of initial body weight, an acceptable functional status would be obtained.

Another study agreeing with our study was done by Christensen et al., (16) who showed that the efficacy of weight reduction on improvement of function in 80 patients with knee OA and showed that weight reduction of $10 \%$ improved knee function by $28 \%$.

Messier et al., (17) concluded that the combination of modest weight loss plus moderate exercise provides better overall improvements in self-reported measures of function and pain and in performance measures of mobility in older overweight and obese adults with knee OA compared with either intervention alone.

A study by Miller et al., (18) suggested that intensive weight loss intervention incorporating energy deficit diet and exercise training improves physical function in older obese adults with knee OA. But they said that greater improvements in function were observed in those with the most weight loss.

Results of the present study were in agreement with meta analysis made by Christensen et al., (15) which indicated that physical disability of patients with knee OA and overweight diminished after a moderate weight reduction regimen. The analysis supported that a weight loss of $>5 \%$ should be achieved within 20 weeks period.

Grundy et al., (19) found that combination of weight loss and exercise provide better improvements in physical function and pain in obese adults with knee OA compared with either intervention alone. Exercise used in association with weight loss provides better improvements in physical capacity and muscle strength

The study of Riddle and Stratford, (20) suggested a dose response relationship between changes in body weight and corresponding changes in pain and function. The threshold for this response gradient appears to be body weight shifts of $\geq 10 \%$. Weight changes of $\geq 10 \%$ have the potential to lead to important changes in pain and function of the knee.

Jenkinson et al., (21) studied the effects of weight loss and quadriceps exercise on 389 men and women aged 45 and over with a body mass index $(\mathrm{BMI}) \geq 28$. They disagreed with this study as they suggested that simple knee strengthening exercises can significantly reduce knee pain and improve knee function in 114 overweight and obese people with knee pain. A moderate sustained weight loss was achieved with dietary intervention but with no apparent influence on pain or function.

At the end of the present study, we found that physical therapy is an effective method of reducing pain and improving function in obese patients with grade I and II of primary knee OA. Weight reduction is also an effective treatment modality in improving pain and knee function in obese patients with grade I and II of primary knee OA. Combination of both physical therapy and weight reduction gives superior results to using either method alone in obese patients with knee OA regarding reduction of disability, improvement of knee pain, physical function, daily activities and quality of life.

\section{References:-}

1. Lopez H.L.,: Nutritional interventions to prevent and treat osteoarthritis. Part I: focus on fatty acids and macronutrients. PMRJ.;2012, 4 (5): S145-154.

2. Diehl P., and Gerdesmeyer L.,: conservative therapy of osteoarthritis. Orthopade, 2013, 42 (2): 125-139.

3. Griffin T.M. and Guilak F., : Why is obesity associated with osteoarthritis? Insights from mouse models of obesity. Biorheology. 2008, 45(3-4): 387-398.

4. Wluka A.E., Lombard C.B., Cicuttini F.M.,: Tackling obesity in knee osteoarthritis. Nat Rev Rheumatol. 2013 ; 9 (4): 225-235.

5. Sridhar M. S., Jarrett C. D., Xerogeanes J. W., et al.,: Obesity and symptomatic osteoarthritis of the knee. J Bone Joint Surg (Br). 2012 ; 94 B (4): 433-40.

6. Dekker J., and Peter W.,: Exercise therapy in hip or knee osteoarthritis. Ned. Tijdschr. Geneeskd. 2011;155 (3031): A3462. 
7. Altman R., Asch E., Bloch D., et al., : Development of criteria for the classification and reporting of osteoarthritis. Classification of osteoarthritis of the knee. Diagnostic and Therapeutic Criteria Committee of the American Rheumatism Association. Arthritis Rheum. 1986; 29 (8): 1039-1049.

8. Guermazi M., Hunter D.J., and Roemer F.W.,: Plain radiography and magnetic resonance imaging diagnostics in osteoarthritis: validated staging and scoring, J Bone Joint Surg Am. 2009; 91 (1): 54-62.

9. Williamson A. and Hoggart B.,: Pain: a review of three commonly used pain rating scales. Journal of Clinical Nursing. 2005; 14: 798-804.

10. Guermazi M, Poiraudeau S, Yahia M, et al,: Translation, adaptation and validation of the Western Ontario and McMaster Universities osteoarthritis index (WOMAC) for an Arab population: the Sfax modified WOMAC. Osteoarthritis and Cartilage. 2004; 12 (6): 459-468.

11. Huang M.H., Chen C.H., Chen T.W., et al., : The effects of weight reduction on the rehabilitation of patients with knee osteoarthritis and obesity. Arthr. Care and Res.2000 ; 13 (6), 398-405.

12. Graeme T, Harding Y, Cheryl L, et al, : Body mass index affects knee joint mechanics during gait differently with and without moderate knee osteoarthritis. Osteoarthritis and Cartilage. 2012; 20: 1234-1242.

13. Fernández C.A, Casariego V.A, Pomar B.,: Comparative study of total energy expenditure estimated by Sense Wear Armband and Harris-Benedict equation in healthy ambulatory population; utility in clinical practice. Nutr. Hosp.2012; 27 (4): 1244-1247.

14. Lee R. and Kean W.F.,: Obesity and knee osteoarthritis. Inflammopharma-cology. 2012; 20 (2): 53-58.

15. Christensen R., Bartels E.M., Astrup A., et al.,: Effect of weight reduction in obese patients diagnosed with knee osteoarthritis: a systematic review and meta -analysis. Ann Rheum Dis. 2007; 66 (4): 433-439.

16. Christensen R., Astrup A., Bliddal D.H., et al.,: Sustained weight loss as a treatment of osteoarthritis in obese patients: long-term results from a randomized trial. Ann. Rheum. Dis.2005; 64 (III): 66.

17. Messier S.P., Loeser R.F., Miller G.D., et al., : Exercise and dietary weight loss in overweight and obese older adults with knee osteoarthritis: the arthritis, diet and activity promotion trial. Arthritis Rheum. 2005; 50: 15011510.

18. Miller G.D., Nicklas B.J., Davis C., et al., : Intensive weight loss program improves physical function in older obese adults with knee osteoarthritis. Obesity (Silver Spring). 2006; 14 (7): 1219-1230.

19. Grundy S.M., Cleeman J.I. and Daniels S.R. : Diagnosis and management of the metabolic syndrome: An American Heart Association. National Heart, Lung, and Blood Institute scientific statement. Curr. Opin. Cardiol. 2006; 21: 1-6.

20. Riddle D.L., and Stratford P.W., : Body weight changes and corresponding changes in pain and function in persons with symptomatic knee osteoarthritis: a cohort study. Arthritis Care Res (Hoboken).2013; 65 (1): 15-22.

21. Jenkinson C.M., Doherty M., Avery A.J., et al., : Effects of dietary intervention and quadriceps strengthening exercises on pain and function in overweight people with knee pain: randomised controlled trial. BMJ. 2009; 18 (339): 3170. 